Review

\title{
Relationship between Aurora-A V57I Polymorphism and the Risk of Cancer: A Meta-Analysis and Trial Sequential Analysis
}

\author{
Guangyuan Chen ${ }^{1 *}$, Cong $\mathrm{Hu}^{1 *}$, Yuxuan Song ${ }^{2 *}$, Mengxi Xiu ${ }^{1}$, Yiling Zhang ${ }^{1}$, Penghui Lai ${ }^{1}$, Yunyan $\mathrm{Li}^{1}$, \\ Xiaoqiang Liu ${ }^{2}$, Peng Huang $3,4 \bowtie$ \\ 1. The Second Clinical Medical School, Nanchang University, Nanchang, Jiangxi 330006, China. \\ 2. Department of Urology, Tianjin Medical University General Hospital, Tianjin 300052, China. \\ 3. Center for Evidence-based Medicine, School of Public Health, Nanchang University, Nanchang 330006, China. \\ 4. Jiangxi Province Key Laboratory of Preventive Medicine, School of Public Health, Nanchang University, Nanchang 330006, China. \\ ${ }^{*}$ Equal contributions and co-first authors.
}

$\triangle$ Corresponding author: Peng Huang, Center for Evidence-based Medicine, School of Public Health, Nanchang University, Nanchang 330006, China. E-mail: pengh@ncu.edu.cn AND huangpengncu@163.com.

(c) The author(s). This is an open access article distributed under the terms of the Creative Commons Attribution License (https://creativecommons.org/licenses/by/4.0/). See http://ivyspring.com/terms for full terms and conditions.

Received: 2019.09.23; Accepted: 2020.01.21; Published: 2020.03.05

\begin{abstract}
Background: It is still conflicting for the correlation between cancer susceptibility and Aurora-A V57I (rs 1047972) gene variant from the published researches. This meta-analysis was performed to access the correlation between cancer susceptibility and Aurora-A rs1047972 gene polymorphism by using meta-analysis methods.

Methods: Eligible studies published before Nov 1, 2019 were systematically searched in PMC, PubMed, EMBASE, Web of Science, Cochrane Library Database, China National Knowledge Infrastructure, Wanfang databases, in order to collect qualified case-control or cohort studies. The odds ratio (OR) and its $95 \%$ confidence interval $(95 \% \mathrm{Cl})$ were used to evaluate the correlation between Aurora-A rs 1047972 gene polymorphism and cancer risk. Sensitivity analysis was used to examine the stability of the results; Egger's test and Begg's funnel chart were used to assess possible publication bias. Trial sequential analysis (TSA) was used to access whether the sample size of our meta-analysis was sufficient.

Results: The sample set extracted from 24 case-control studies involving 35,926 subjects $(14,639$ cases and 21,287 controls) for the association of Aurora-A rs1047972 gene polymorphism with cancer susceptibility. In our meta-analysis, Aurora-A rs 1047972 polymorphism was associated with an increased risk of cancer susceptibility in overall populations (GA+GG vs. AA: $P=0.039$, OR=1.106; $95 \% \mathrm{Cl} 1.005-$ 1.218; $A A$ vs. $G G: P=0.003, O R=0.814 ; 95 \% \mathrm{Cl}, 0.710-0.934)$, and the $G A / G G$ variant might be a risk factor for cancer susceptibility. In the stratified analysis by ethnicity, we found a significant association between Aurora-A rs 1047972 variant and the susceptibility of the cancer in Caucasian population. In a subgroup analysis by cancer type, we observed a significantly increased susceptibility of lung cancer. In addition, an increased risk was found between Aurora-A rs 1047972 polymorphism and cancer susceptibility in MALDI-TOF group and among population-based study (PB) patients. Our results were in a sufficiently large number of participants according to TSA and did not require more studies to confirm such association.

Conclusion: Our meta-analysis revealed that the susceptibility of cancer was associated with Aurora-A rs 1047972 polymorphism, especially in Caucasians. And the GA/GG variant might be a risk factor for cancer susceptibility.
\end{abstract}

Key words: Aurora-A, polymorphism, meta-analysis, cancer 


\section{Introduction}

Aurora Kinase A (AURKA) is a serine/threonine kinase, which belongs to the Aurora kinase family [1]. In the process of mitosis, AURKA plays an important role in the cell cycle by participating in the separation and maturation of the centrosome and the establishment of the bipolar mitotic spindles, ensuring the correct separation of chromosomes and the completion of cytoplasmic division [2]. Abnormal amplification and high expression of AURKA are common in a variety of gastric adenocarcinomas and esophageal adenocarcinomas [3]. Recent studies have suggested that AURKA can participate in some important cell signaling pathways such as the Wnt signaling pathway. It directly or indirectly regulates the expression of some important proteins, and has extraordinary impact on the development of tumors [4-5].

The AURKA gene owns a lot of polymorphisms, so far, at least 6 AURKA polymorphism sites have been found, of which the most investigated were two common non-synonymous single nucleotide polymorphisms (SNPs) (F31I and V57I). However, there were at least four meta-analyses indicating F31I SNP was a low protective factor in the development of tumors. So we only analyzed the SNP V57I and did not analyze SNP F31I, as we needed to be sure that there was no redundancy that may be caused out of the publication of the research for SNP F31I. Gene polymorphism has been reported to be a significant factor and it might increase the susceptibility of cancer [6-7]. At present, more and more epidemiologic researches are centered on correlation between rs1047972 (V57I) gene polymorphism and cancers risk, but the conclusions are still controversial. Based on it, this meta-analysis was performed to investigate whether the Aurora-A rs1047972 (V57I) gene polymorphism was correlated with the susceptibility of cancer by widely collecting the reported investigations.

\section{Materials and Methods}

\section{Literature retrieval}

Eligible studies were systematically searched in PMC, Cochrane Library database, PubMed, EMBASE, Web of Science, China National Knowledge Infrastructure, Wanfang databases. And we also performed manual search to find relevant studies. English search strategy: (Aurora-A OR BTAK OR STK15 OR AIKI OR rs1047972) AND (cancer OR neoplasm OR tumor OR carcinoma) AND (SNP or variant or genotype or polymorphism). We searched the studies published before Nov 1, 2019, and also searched relevant degree papers.

\section{Inclusion and exclusion criteria}

Inclusion criteria

(1) Case-control or cohort studies on the association between Aurora-A rs1047972 gene polymorphism and cancers risk; (2) The genotypes of the study followed with Hardy-Weinberg equilibrium (HWE); (3) Alleles or genotypes frequencies in control groups and case groups could be received from the studies; (4) English and Chinese literature.

\section{Exclusion criteria}

(1) Unable to extract useful data or results; (2) The Aurora-A rs1047972 polymorphism was not included or the results were not about cancers susceptibility; (3) The studies published repeatedly and the one included the largest sample size.

\section{Data extraction and literature quality evaluation}

Using the Newcastle-Ottawa Scale (the NOS scale) to evaluate the included articles, if the score of the articles were more than or equal to 5 points, the quality of the articles was considered to be high. Two authors independently read the retrieved article titles and abstracts. After excluding the articles that did not meet the inclusion criteria, the full review of the retrieved articles probably meeting the inclusion criteria was performed to determine its qualifications for final incorporation after cross-checking by the two research evaluators. The extraction information mainly includes: the name of the first author of the studies, ethnicity, original country, the year of publication, sample size, source of controls (population-based controls and hospital-based controls), genotyping methods, the number of cases and controls for Aurora-A rs1047972 genotypes and so on.

\section{Statistical analyses}

We performed meta-analysis using STATA 12.0 software, and the count data was expressed using odds ratio (OR) and its 95\% confidence interval (95\% $\mathrm{CI})$. The heterogeneity between the results of each included study was tested by chi-square test. When there was a small statistical homogeneity between these studies ( $P>0.1$ and $I^{2}<50 \%$ ), a meta-analysis was performed by using a fixed-effect model; if there was a huge statistical heterogeneity between these studies $\left(\mathrm{P}<0.1\right.$ or $\left.I^{2}>50 \%\right)$, we analyzed the results using a random-effect model, if necessary, a subgroup analysis of related factors that may lead to heterogeneity will be performed. The pooled ORs were performed for these models (1) AA vs.GG, (2) GA vs. AA, (3) AA+GA vs.GG and (4) GG+GA vs. 
AA, respectively. We use Egger's test and Begg's funnel chart to evaluate the publication of bias. Each time a document was removed for sensitivity analysis. Subgroup analysis was carried out, as it needed.

\section{Trial Sequential Analysis (TSA)}

We used the TSA v0.9.5.10 Beta software to perform the trial sequential analysis. Our study sets the odds ratio reduction to $20 \%$, the first type of error $\alpha=0.05$, and the second type of error $\beta=0.2$ to evaluate the required information size (RIS). At the same time, if the cumulative $Z$ value crosses the RIS threshold, the results are considered statistically significant. Therefore, it can be considered the sample size of the accumulated evidence is sufficient. However, if the cumulative $Z$ value does not cross the RIS threshold, it means the sample size is not sufficient. And it still needs more studies to confirm the results.

\section{Results}

\section{Characteristics of the selected studies}

The search strategy resulted in a total of 425 potentially relevant articles (Figure 1). The characteristics of the included studies were listed in Table 1. For Aurora-A V57I (rs1047972) polymorphism, 22 articles [8-29] were investigated. However, there were three independent groups in Dicioccio's study [14], and we treated them separately. Finally, 24 case-control studies were included in the present meta-analysis. Of these 24 studies (including 14,639 cases and 21,287 controls), seven studied the association between Aurora-A rs1047972 variant and the risk of breast cancer, three studied the association between Aurora-A rs1047972 variant and the susceptibility of ovarian cancer and three studied lung cancer. The other studied the susceptibility of bladder, cervical, gastric and colorectal cancer. As far as the genotyping methods, 8 were using PCR, 11 were using TaqMan, 4 were using PCR-RFLP, and one was using MALDI-TOF. There are 10 studies for the Asian populations and 14 studies for the Caucasian populations. The NOS scores of the 24 documents were all more than 5 it means that all of them were high quality studies (Table 1; Figure 1).

\section{Meta-analysis results}

Association between the risk of cancer and Aurora-A rs 1047972 polymorphism in the total population

24 case-control studies including 14,639 cancer cases and 21,287 normal controls were investigated. As illustrated in Table 2, a significant increased risk was observed for Aurora-A rs1047972 gene polymorphism and cancer susceptibility in overall population (GA+GG vs. AA: $\mathrm{P}=0.039, \mathrm{OR}=1.106,95 \%$ CI 1.005-1.218; AA vs. GG: $P=0.003, \mathrm{OR}=0.814,95 \%$ CI $0.710-0.934)$. And the AA genotype carriers have a slightly lower incidence of cancer compared to that of GG or GA carriers and the GG or GA variant might be a risk factor for cancer susceptibility in overall populations (Table 2; Figure 2).

Table 1. Main characters of studies included in this meta-analysis

\begin{tabular}{|c|c|c|c|c|c|c|c|c|c|}
\hline First author & Year & Country & Ethnicity & Cancer type & $\mathrm{SC}$ & Case(n) & Control(n) & GM & NOS score \\
\hline Egan & 2004 & USA & Caucasian & Breast cancer & PB & 905 & 788 & PCR & 8 \\
\hline Dicioccio a & 2004 & UK & Caucasian & Ovarian cancer & PB & 750 & 843 & TaqMan & 7 \\
\hline Dicioccio b & 2004 & USA & Caucasian & Ovarian cancer & PB & 323 & 427 & TaqMan & 7 \\
\hline Dicioccio c & 2004 & Denmark & Caucasian & Ovarian cancer & PB & 432 & 1112 & TaqMan & 7 \\
\hline Dai & 2004 & China & Asian & Breast cancer & $\mathrm{PB}$ & 1102 & 1186 & TaqMan & 8 \\
\hline Lo & 2005 & China & Asian & Breast cancer & $\mathrm{HB}$ & 704 & 1950 & TaqMan & 8 \\
\hline Kimura & 2005 & Japan & Asian & Esophageal cancer & $\mathrm{HB}$ & 197 & 146 & PCR & 7 \\
\hline Hienonen & 2006 & Finland & Caucasian & Colorectal cancer & $\mathrm{HB}$ & 125 & 94 & PCR & 8 \\
\hline Cox & 2006 & USA & Caucasian & Breast cancer & $\mathrm{PB}$ & 1240 & 1724 & TaqMan & 9 \\
\hline $\mathrm{Ju}$ & 2006 & Korea & Asian & Gastric cancer & $\mathrm{HB}$ & 501 & 427 & PCR & 8 \\
\hline $\mathrm{Gu}$ & 2007 & USA & Caucasian & Lung cancer & $\mathrm{HB}$ & 1098 & 1027 & TaqMan & 9 \\
\hline Chen & 2007 & USA & Caucasian & Colorectal cancer & $\mathrm{HB}$ & 60 & 65 & PCR & 6 \\
\hline Milam & 2007 & USA & Caucasian & Cervical cancer & $\mathrm{HB}$ & 140 & 188 & TaqMan & 7 \\
\hline Wang & 2007 & USA & Caucasian & Lung cancer & $\mathrm{HB}$ & 1263 & 1154 & TaqMan & 7 \\
\hline Ye & 2008 & USA & Caucasian & Bladder cancer & $\mathrm{HB}$ & 604 & 593 & TaqMan & 9 \\
\hline Dogan & 2008 & Turkey & Caucasian & Lung cancer & $\mathrm{HB}$ & 102 & 102 & PCR & 8 \\
\hline Guénard & 2009 & Canada & Caucasian & Breast cancer & $\mathrm{HB}$ & 96 & 96 & PCR & 7 \\
\hline MARIE-GENICA & 2010 & German & Caucasian & Breast cancer & PB & 3139 & 5469 & MALDI-TOF & 9 \\
\hline Lai & 2018 & Malaysia & Asian & Gastric cancer & PB & 41 & 1110 & PCR-RFLP & 6 \\
\hline Eric & 2017 & Malaysia & Asian & Breast cancer & $\mathrm{HB}$ & 71 & 260 & PCR-RFLP & 6 \\
\hline Bao & 2017 & China & Asian & Hepatocellular carcinoma & $\mathrm{HB}$ & 348 & 359 & PCR-RFLP & 9 \\
\hline Chou & 2019 & China & Asian & Oral cancer & $\mathrm{HB}$ & 876 & 1200 & PCR & 8 \\
\hline Huang a & 2019 & China & Asian & Urothelial Cell Carcinoma & $\mathrm{HB}$ & 431 & 862 & TaqMan & 7 \\
\hline Huang $b$ & 2019 & China & Asian & Oral cancer & $\mathrm{HB}$ & 91 & 105 & PCR-RFLP & 8 \\
\hline
\end{tabular}

Abbreviations: PCR, polymerase chain reaction; RFLP, restriction fragment length polymorphism; SC, source of control; GM, genotype methods; HB, hospital-based study; PB, population-based study; MALDI-TOF: Matrix-Assisted Laser Desorption/Ionization Time of Flight Mass spectrometry; NA, not available. 


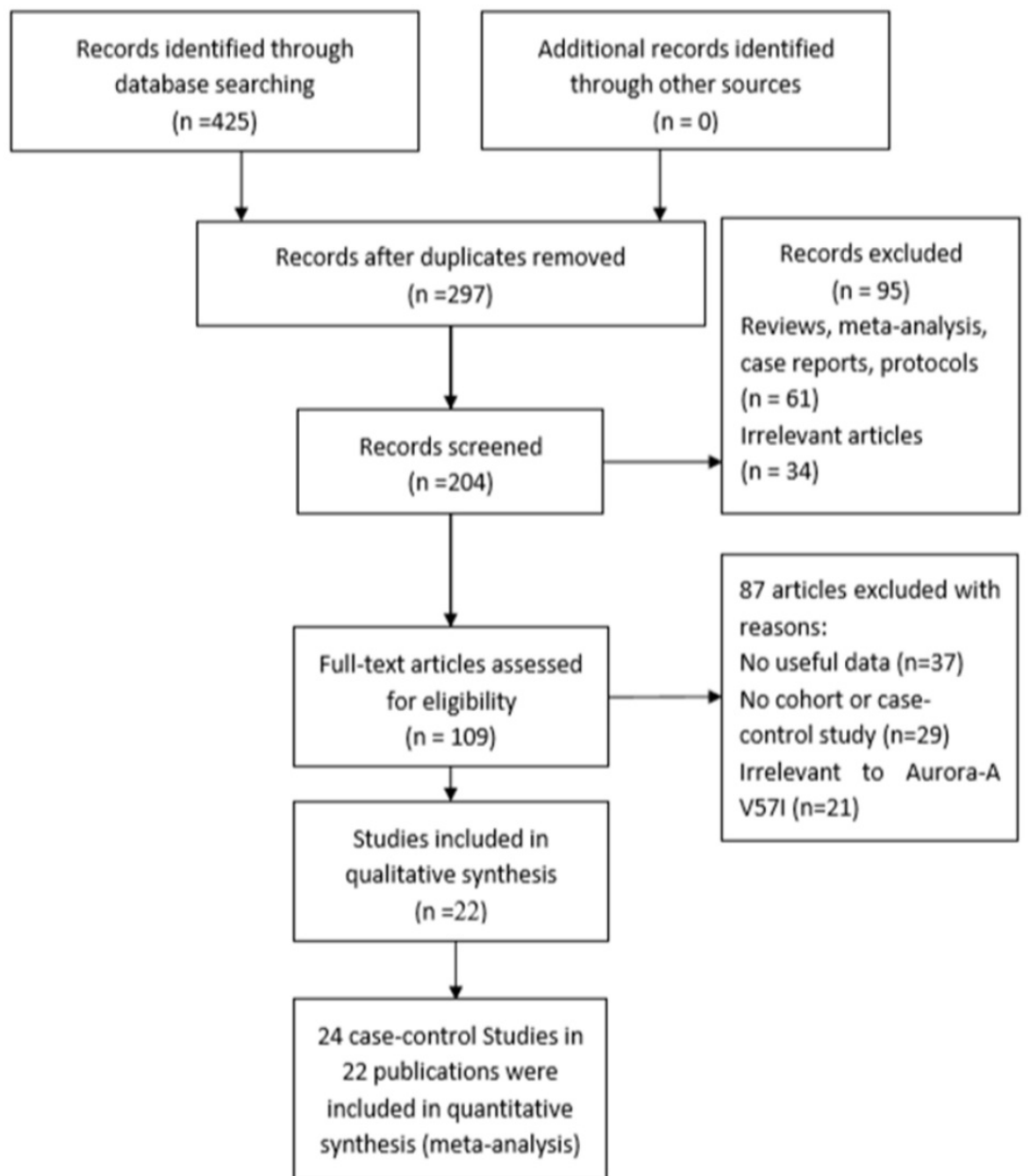

Figure 1. Flowchart illustrating the search strategy for Aurora-A V57I variant and the risk of cancer.

Table 2. Meta-analysis results

\begin{tabular}{|c|c|c|c|c|c|c|c|c|}
\hline \multirow[t]{2}{*}{ Study } & \multirow[t]{2}{*}{ Contrast model } & \multirow[t]{2}{*}{ OR $(95 \% \mathrm{Cl})$} & \multirow[t]{2}{*}{$\mathrm{P}$} & \multicolumn{2}{|c|}{ Test for heterogeneity } & \multicolumn{2}{|c|}{ Publication bias (Egger's test) } & \multirow[t]{2}{*}{ Analysis model } \\
\hline & & & & $\mathrm{I}^{2}(\%)$ & $\mathrm{P}$ & $t$ & $\mathrm{P}$ & \\
\hline \multirow[t]{4}{*}{ Overall } & AA vs.GG & $0.814(0.710,0.934)$ & 0.003 & $0.00 \%$ & 0.535 & 1.49 & 0.150 & F \\
\hline & GA vs.AA & $1.079(0.977,1.191)$ & 0.134 & $13.20 \%$ & 0.279 & -0.02 & 0.987 & $\mathrm{~F}$ \\
\hline & $\mathrm{AA}+\mathrm{GA}$ vs.GG & $0.960(0.912,1.010)$ & 0.118 & $3.50 \%$ & 0.413 & -0.06 & 0.952 & $\mathrm{~F}$ \\
\hline & GG+GA vs.AA & $1.106(1.005,1.218)$ & 0.039 & $18.30 \%$ & 0.210 & -0.19 & 0.854 & $\mathrm{~F}$ \\
\hline \multirow[t]{4}{*}{ Asian } & AA vs.GG & $0.872(0.662,1.150)$ & 0.333 & $15.40 \%$ & 0.301 & & & $\mathrm{~F}$ \\
\hline & GA vs.AA & $0.986(0.868,1.121)$ & 0.833 & $22.80 \%$ & 0.233 & & & $\mathrm{~F}$ \\
\hline & $\mathrm{AA}+\mathrm{GA}$ vs.GG & $0.906(0.736,1.116)$ & 0.353 & $39.90 \%$ & 0.092 & & & $\mathrm{R}$ \\
\hline & GG+GA vs.AA & $1.017(0.898,1.151)$ & 0.793 & $30.30 \%$ & 0.166 & & & $\mathrm{~F}$ \\
\hline \multirow[t]{4}{*}{ Caucasian } & AA vs.GG & $0.797(0.681,0.933)$ & 0.005 & $0.00 \%$ & 0.636 & & & $\mathrm{~F}$ \\
\hline & GA vs.AA & $1.234(1.054,1.445)$ & 0.009 & $0.00 \%$ & 0.666 & & & $\mathrm{~F}$ \\
\hline & $\mathrm{AA}+\mathrm{GA}$ vs.GG & $0.952(0.900,1.008)$ & 0.090 & $0.00 \%$ & 0.819 & & & $\mathrm{~F}$ \\
\hline & GG+GA vs.AA & $1.254(1.077,1.461)$ & 0.004 & $0.00 \%$ & 0.609 & & & $\mathrm{~F}$ \\
\hline
\end{tabular}

Abbreviations: R: random-effect model; F: fixed-effect model; OR: odds ratio; CI: confidence interval.

Association between cancer risk and Aurora-A rs 1047972 polymorphism in subgroup analysis by ethnicity

Subgroup analysis of different ethnicities was carried out. We found a significant association between Aurora-A rs1047972 variant and the susceptibility of the cancer in Caucasian population (AA vs. GG: $\mathrm{P}=0.005$, OR=0.797, 95\% CI 0.681-0.933;
GA vs. AA: $P=0.009, \mathrm{OR}=1.234,95 \%$ CI $1.054-1.445$; GA+GG vs. AA: $\mathrm{P}=0.004, \mathrm{OR}=1.254,95 \%$ CI 1.077 1.461). Our results indicated that GA/GG genotype carriers have a higher risk of cancer compared to AA carriers and the AA variant might be a protective factor for cancer susceptibility in Caucasian population. However, we did not find any association between Aurora-A rs1047972 variant and cancer risk in Asian population (Table 2). 
A

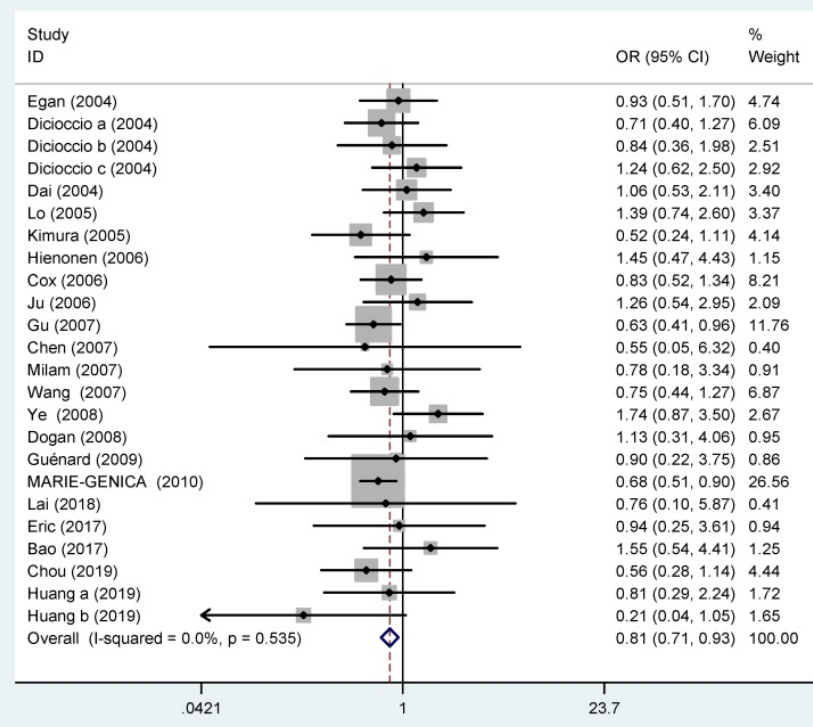

C

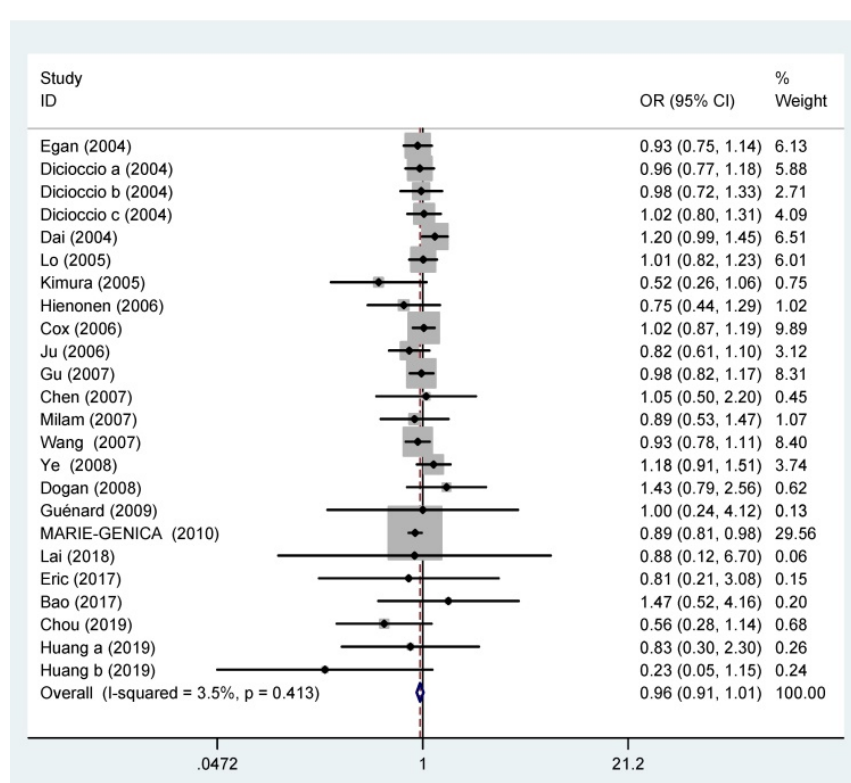

B

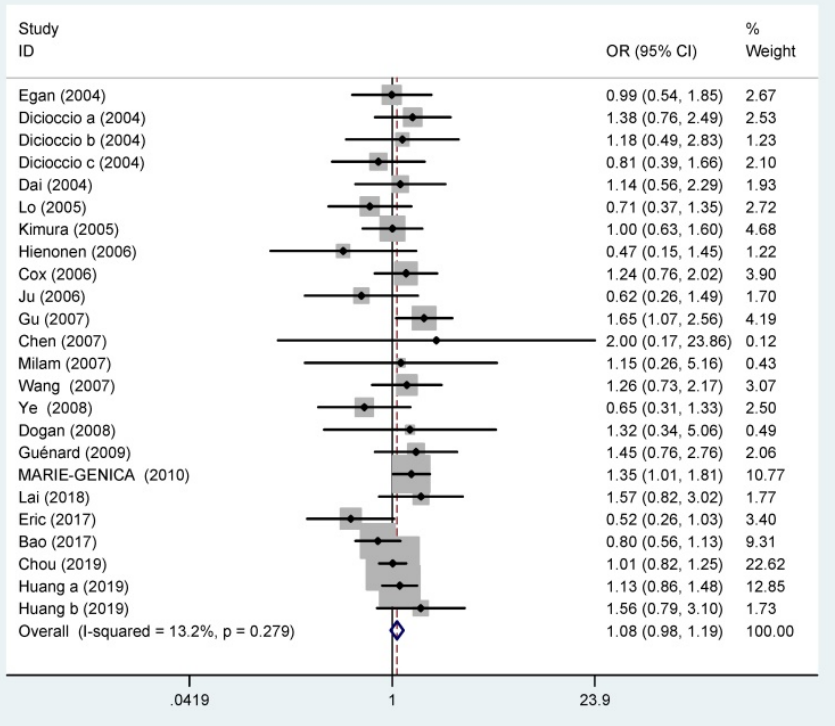

D

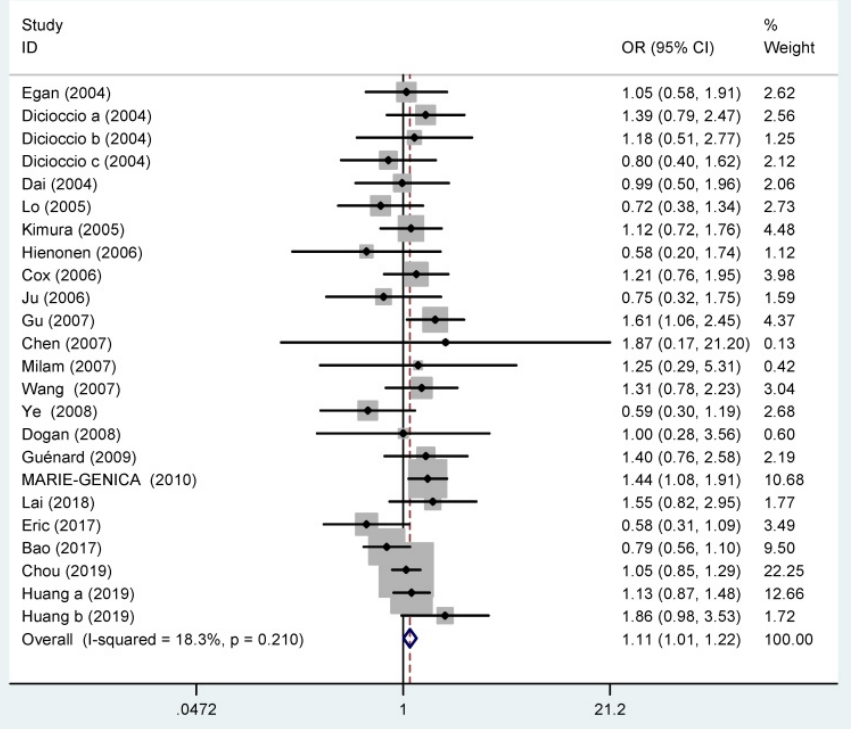

Figure 2. Forest plot of association between Aurora-A V57l variant and cancer (A: AA vs.GG; B: GA vs. AA; C: AA+GA vs.GG; D: GG+GA vs. AA).

\section{Association between Aurora-A rs 1047972 polymorphism and cancer risk in subgroup analysis by cancer type}

In a stratified analysis by different types of cancer, our results suggested that Aurora-A rs1047972 variant has an increased risk for lung cancer susceptibility ( $\mathrm{GA}+\mathrm{GG}$ vs. AA: $\mathrm{P}=0.022, \mathrm{OR}=1.451$, 95\% CI 1.056-1.994). And the AA variant might be a protective factor in lung cancer. But for the other cancer, like breast cancer and cervical cancer, we did not find any important association in different Aurora-A rs1047972 genotype carriers (Table 3).
Association between Aurora-A rs 1047972

polymorphism and cancer risk in subgroup analysis by source of control (SC) and genotype methods (GM)

In a stratified analysis by SC, we found an increased risk of cancer risk in $\mathrm{PB}$ (population-based study) group (GA+GG vs. AA: $P=0.008, O R=1.274$, 95\% CI 1.064-1.525). Our results indicated that GA / GG genotype carriers have a higher risk of cancer compared to that of AA carriers and the AA variant might be a protective factor for cancer susceptibility in PB group. However, we did not find any association between Aurora-A V57I variant and cancer risk in HB (hospital-based study) group (Table 3). 
Table 3. Subgroup of Meta-analysis results

\begin{tabular}{|c|c|c|c|c|c|c|c|}
\hline \multirow[t]{2}{*}{ Study } & \multirow[t]{2}{*}{ Subgroup } & \multirow[t]{2}{*}{ Contrast model } & \multirow[t]{2}{*}{ OR $(95 \% \mathrm{Cl})$} & \multirow[t]{2}{*}{$\mathrm{P}$} & \multicolumn{2}{|c|}{ Test for heterogeneity } & \multirow[t]{2}{*}{ Analysis model } \\
\hline & & & & & $\mathrm{I}^{2}(\%)$ & $\mathrm{P}$ & \\
\hline \multirow[t]{11}{*}{ Cancer type } & Lung cancer & GG+GA vs.AA & $1.451(1.056,1.994)$ & 0.022 & $0.00 \%$ & 0.706 & F \\
\hline & Breast cancer & GG+GA vs.AA & $1.153(0.962,1.382)$ & 0.123 & $38.90 \%$ & 0.132 & $\mathrm{~F}$ \\
\hline & Ovarian cancer & GG+GA vs.AA & $1.139(0.768,1.687)$ & 0.518 & $0.00 \%$ & 0.487 & $\mathrm{~F}$ \\
\hline & Esophageal cancer & GG+GA vs.AA & $1.123(0.716,1.763)$ & 0.613 & NA & NA & $\mathrm{F}$ \\
\hline & Colorectal cancer & GG+GA vs.AA & $0.713(0.271,1.878)$ & 0.494 & $0.00 \%$ & 0.389 & $\mathrm{~F}$ \\
\hline & Gastric cancer & GG+GA vs.AA & $1.173(0.704,1.955)$ & 0.541 & $44.80 \%$ & 0.178 & $\mathrm{~F}$ \\
\hline & Oral cancer & GG+GA vs.AA & $1.282(0.750,2.190)$ & 0.364 & $63.80 \%$ & 0.096 & $\mathrm{R}$ \\
\hline & Cervical cancer & GG+GA vs.AA & $1.248(0.293,5.311)$ & 0.765 & NA & NA & $\mathrm{F}$ \\
\hline & Bladder cancer & GG+GA vs.AA & $0.593(0.296,1.188)$ & 0.141 & NA & NA & $\mathrm{F}$ \\
\hline & Hepatocellular carcinoma & GG+GA vs.AA & $0.786(0.560,1.103)$ & 0.164 & NA & NA & $\mathrm{F}$ \\
\hline & Urothelial Cell Carcinoma & GG+GA vs.AA & $1.132(0.868,1.478)$ & 0.360 & NA & NA & $\mathrm{F}$ \\
\hline \multirow[t]{2}{*}{ SC } & $\mathrm{PB}$ & GG+GA vs.AA & $1.274(1.064,1.525)$ & 0.008 & $0.00 \%$ & 0.800 & $\mathrm{~F}$ \\
\hline & $\mathrm{HB}$ & GG+GA vs.AA & $1.044(0.932,1.170)$ & 0.454 & $29.10 \%$ & 0.132 & $\mathrm{~F}$ \\
\hline \multirow[t]{4}{*}{ GM } & PCR & GG+GA vs.AA & $1.053(0.894,1.241)$ & 0.535 & $0.00 \%$ & 0.894 & F \\
\hline & TaqMan & GG+GA vs.AA & $1.137(0.973,1.327)$ & 0.105 & $0.30 \%$ & 0.437 & F \\
\hline & MALDI-TOF & GG+GA vs.AA & $1.436(1.082,1.906)$ & 0.012 & NA & NA & $\mathrm{F}$ \\
\hline & PCR-RFLP & GG+GA vs.AA & $1.041(0.632,1.714)$ & 0.875 & $70.00 \%$ & 0.019 & $\mathrm{~F}$ \\
\hline
\end{tabular}

Abbreviations: R: random-effect model; F: fixed-effect model; OR: odds ratio; CI: confidence interval; SC, source of control; GM, genotype methods; HB, hospital-based study; PB, population-based study; NA, not available.

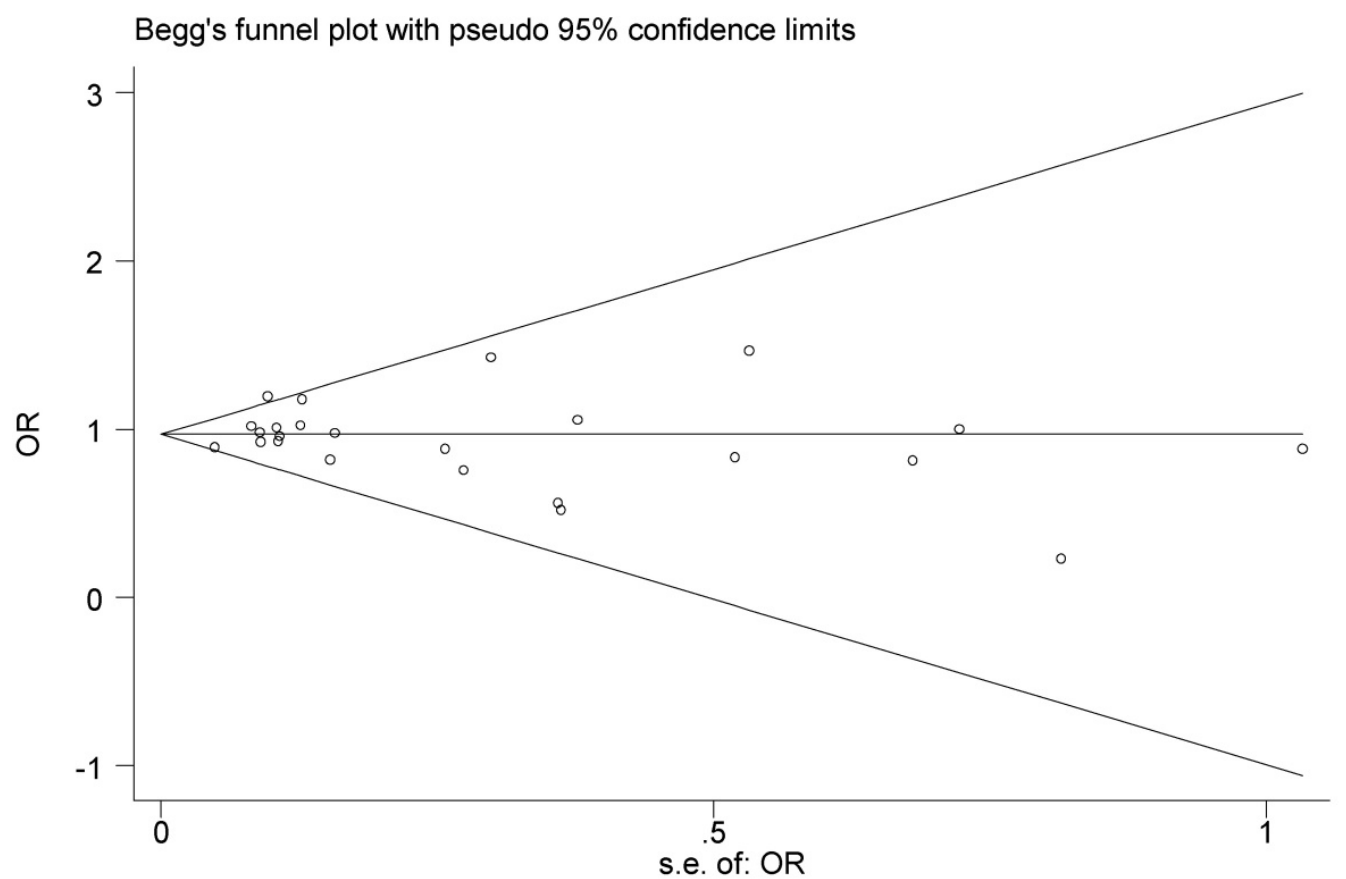

Figure 3. Begg's funnel plot with pseudo $95 \%$ confidence limits for studies of the association between cancer risk and Aurora-A V57I variant (the dominant model).

In a stratified analysis by GM, we observed a significant association between Aurora-A rs1047972 variant and increased risk of cancer in MALDI-TOF group (GA+GG vs. AA: $\mathrm{P}=0.012$, $\mathrm{OR}=1.436,95 \% \mathrm{CI}$ 1.082-1.906). Our results indicated that GA/GG genotype carriers have a higher risk of cancer compared to that of AA carriers (Table 3).

\section{Sensitivity analysis}

In order to evaluate the stability of the analysis results, we conducted a sensitivity analysis by removing each individual research from the analysis at a time. It showed that the changes of each genetic contrast model results were not obvious, suggesting that the results of meta-analysis were stable and reliable.

\section{Publication bias}

We assessed the publication bias by Begg's funnel plots and Egger's test (dominant model: Egger's test $P=0.952)$, and the results suggesting that there was no publication bias for the association between Aurora-A rs1047972 gene polymorphism and cancers risk in included studies (Figure 3). 
TSA is a Two-sided graph

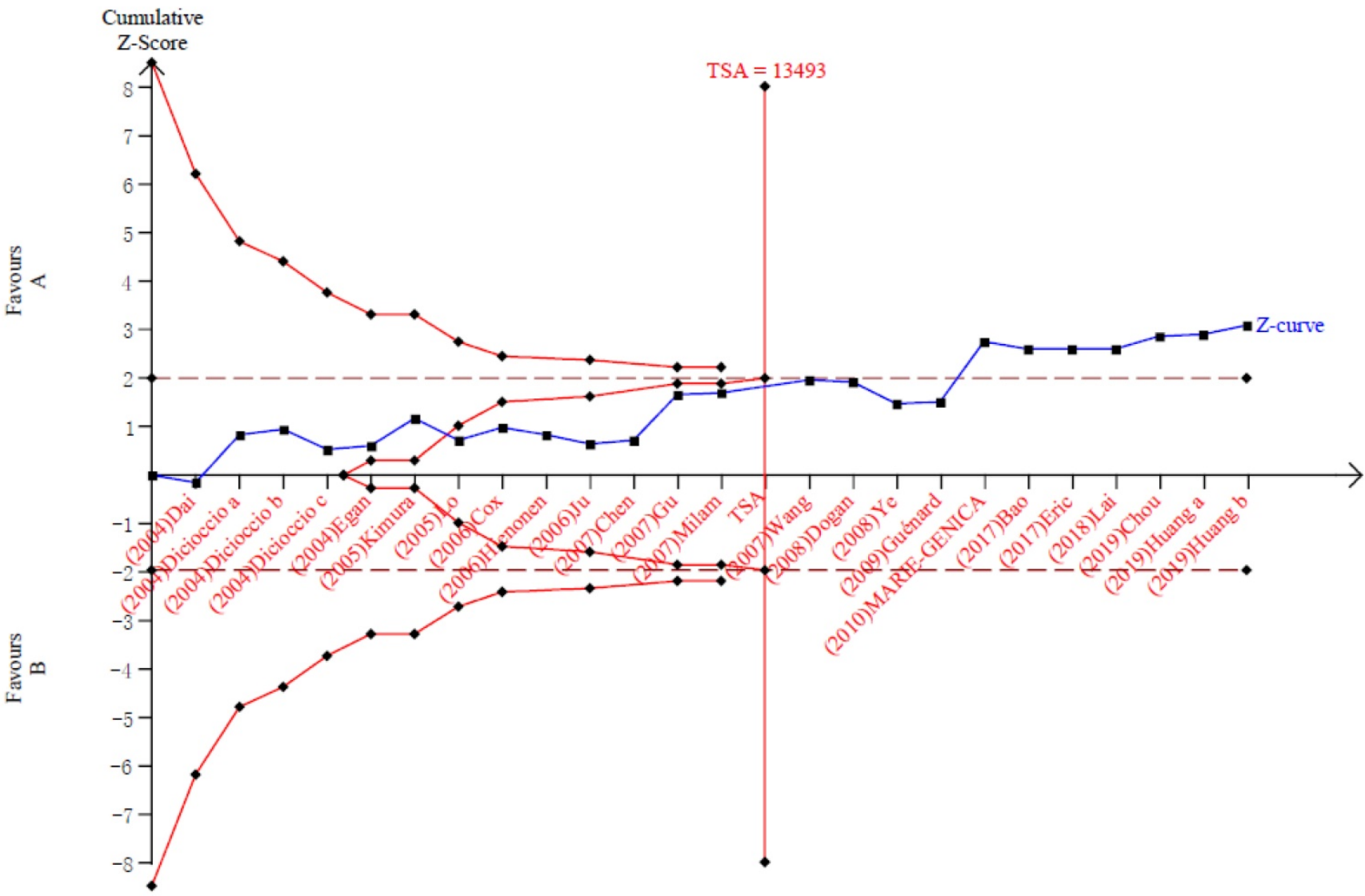

Figure 4. Trial sequential analysis of the association between Aurora-A V57I variant and the risk of cancer. The required information size was calculated based on a two side $\alpha=$ $5 \%, \beta=15 \%$ (power $80 \%$ ), and a relative risk reduction of $20 \%$.

\section{Trial Sequential Analysis results}

We implemented TSA to reduce the risk of type I error by keeping the overall $5 \%$ risk of a type I error and $20 \%$ risk of a type II error (power of $80 \%$ ) to evaluate the RIS. As it showed, the sample size of the 18th study had crossed the TSA boundary (Figure 4). The positive conclusion was obtained in advance, which was consistent with the above meta-analysis results. And the sample size had reached the required information size $(13,493$ cases $)$ to obtain a positive conclusion. Therefore, it can be thought that AA carriers have a lower risk of cancers than GG carriers, and the evidence is reliable. The actual result was shown in Figure 4.

\section{Discussion}

In the past 20 years, a large amount of epidemiologic researches were centered on correlation between Aurora-A rs1047972 gene polymorphism and cancers risk, but the conclusions are still controversial. Based on it, this meta-analysis was performed to investigate whether the Aurora-A rs1047972 gene polymorphism was correlated with the susceptibility of cancer. In this meta-analysis, a total of 24 case-control studies including 14,639 cancer cases and 21,287 normal controls were investigated. Our results demonstrated that a significant association was observed for Aurora-A rs1047972 gene polymorphism in overall population. And the AA genotype carriers have a slightly lower incidence of cancer compared to that of GA/GG carriers and the GA/GG variant might be a risk factor for cancer susceptibility in overall populations.

In a stratified analysis by population, we found a significant association between Aurora-A rs1047972 variant and the susceptibility of the cancer in Caucasian population. Our results indicated that GA/ GG genotype carriers have a higher risk of cancer compared to that of AA carriers and the AA variant might be a protective factor for cancer susceptibility in Caucasian population. However, we did not find any association between Aurora-A rs1047972 variant and cancer risk in Asian population. In a stratified analysis by cancer type, our results indicated that Aurora-A rs1047972 polymorphism has an increased risk for lung cancer.

Our results were consistent with the study by $\mathrm{Gu}$ et al. [7]. Because Gu et al. also found that Aurora-A 
rs1047972 polymorphism has an increased risk for lung cancer. But our results were different from Lai et al. [24] and Bao et al. [26], Lai et al. found the association of Aurora-A rs1047972 polymorphism with an increased risk of gastric cancer susceptibility in Malaysians, and gastric cancer incidences among Malaysians have significantly association among younger age group ( $<50$ years). Bao et al. found AA carriers of the Aurora-A rs1047972 polymorphism were significantly associated with decreased susceptibility to HBV-related hepatocellular carcinoma when compared with non-carriers. However, we did not find any significantly association in gastric cancer and hepatocellular carcinoma susceptibility. In addition, Chen et al. [23] found that the Aurora-A rs1047972 polymorphism was associated with protection from colorectal cancer susceptibility. Why the studies above have the different results, the possible reasons might illustrate as below: First, cancer is a complex disease, and various environmental factors might have an influence on the susceptibility of different cancers. During the occurrence and development of malignant tumors, mutations of various tumor suppressor genes and activation of oncogenes are important starting and promoting factors, and they run through the entire process of tumor progression. Second, the difference of ethnic (Asians and Caucasians) in the Aurora-A rs1047972 gene frequencies might also lead to these different results. Third, the other gene polymorphisms might have an effect on the cancer susceptibility, not only the Aurora-A rs1047972 polymorphism. In addition, the increase of various cytokines, chemokines and inflammatory mediators can also promote angiogenesis, cause suppression of local immunity and inhibit apoptosis, and can increase the risk of tumor metastasis.

We also carried out subgroup analysis by SC and GM. In a stratified analysis by SC, we found significant association between Aurora-A rs1047972 polymorphism and increased risk of $\mathrm{PB}$ group. In a stratified analysis by GM, we observed significant association between Aurora-A rs1047972 polymorphism and increased risk of MALDI-TOF group. Our results indicated that different genotype methods might have an important affect in different studies.

A previous meta-analysis conducted by Tang et al. [30] included 14 case-control studies. They found Aurora-A rs1047972 polymorphism was a protective factor in Caucasians. However, based on more studies, we found Aurora-A rs1047972 polymorphism was a risk factor in Caucasians. There are some reasons for the different results between Tang et al. and our study: First, the number of patients included in Tang's study was relatively small, and Tang's study was published in 2014, there were many case-control studies in the past five years been published. With more included studies and larger sample sizes, our findings and our results were more accurate and reliable. Second, compared with Tang's study, we also analyzed the relationship between more types of cancer risk and genetic polymorphisms such as cervical cancer, esophageal cancer and hepatocellular carcinoma, which could provide more clues and guidance for clinically practical work. In addition, huge heterogeneity was observed in Tang's study $\left(\mathrm{I}^{2}=\right.$ $55.4 \%)$ but not in our meta-analysis $\left(\mathrm{I}^{2}=0 \%\right)$, so this might distort their results.

It is very important for us to pay more attention to the heterogeneity, because huge heterogeneity might have the bad influence on our results of the present meta-analysis and neglect the heterogeneity might lead to mistakes. In the present meta-analysis, the contrast model of Aurora-A rs1047972 polymorphism did not have significant heterogeneity. Therefore, it can be considered the results we performed were reliable in a way. In addition, we carried out the subgroup analysis of other related factors in order to study the relationship between more factors and cancer susceptibility.

Moreover, our meta-analysis has some advantages. Firstly, we have carried out TSA to reduce the article risk of type I error to promote scientific preciseness. Secondly, we have revealed a lot of factors which exert influence on the association between Aurora-A rs1047972 polymorphism and the susceptibility to cancer. Thirdly, strictly based on the search strategy, articles included in our meta-analysis are more than 20 studies which may enhance the authenticity and reliability of the analysis. Fourthly, the subgroup analysis is sufficient and reveals the difference among diverse populations. In addition, Egger's tests and funnel plot were used to find potential publication bias. No significant publication bias was found.

Nevertheless, there are still some limitations which cannot be avoided in our present meta-analysis. First, some unpublished studies might not be included. Second, we did not include studies in other races such as Africa, so it cannot fully reflect the influence of different races and regions. Third, this study did not reveal gene environment and gene-gene interactions, due to the original information of the included studies was not sufficient. Fourth, this study failed to include an independent cohort study into our meta-analysis; therefore, more cohort studies should be performed to refute the association between the risk of cancer and Aurora-A rs1047972 polymorphism in the future. Despite the above limitations, this study 
ensures the reliability of the results by setting a careful research plan to minimize the bias.

\section{Conclusion}

In summary, the findings from this systematic review and meta-analysis indicate that the susceptibility of cancer was associated with Aurora-A rs1047972 polymorphism, especially, in Caucasians. And the AA genotype carriers have a slightly lower incidence of cancer compared to that of GA/GG carriers and the GA/GG variant might be a risk factor for cancer susceptibility. Our study has certain guiding significance for Aurora-A rs1047972 polymorphism and cancer research, and contributes to the development of ARUKA inhibitors. Of course, due to the research we included in this meta-analysis was the only case-control study, more prospective study should be performed to refute the association between the risk of cancer and Aurora-A rs1047972 polymorphism in the future.

\section{Abbreviations}

OR: odds ratio; CI: confidence interval; HWE: Hardy-Weinberg equilibrium; AURKA: Aurora-A; SNP: single nucleotide polymorphism; the NOS scale: the Newcastle-Ottawa Scale; R: random-effect model; F: fixed-effect model; SC: source of control; GM: genotype methods; PCR: polymerase chain reaction; RFLP: restriction fragment length polymorphism; HB: hospital-based study; PB: population-based study.

\section{Acknowledgements}

\section{Author Contributions}

Study design with methodology checking: Peng Huang, Xiaoqiang Liu, Yuxuan Song, Guangyuan Chen and Cong Hu; Data analysis guidance: Peng Huang, Xiaoqiang Liu, and Yuxuan Song; Literature screening: Yuxuan Song, Penghui Lai, Yiling Zhang, Yunyan Li and Mengxi Xiu; Data collection: Guangyuan Chen, Cong Hu, Penghui Lai, Yiling Zhang; Data analysis: Guangyuan Chen, Cong Hu; Writing: Guangyuan Chen, Cong $\mathrm{Hu}$ and Yuxuan Song. Grammar check: Yuxuan Song, Guangyuan Chen and Cong $\mathrm{Hu}$.

We thank Haifei Zhang, Junzhe Liu, Zhou Xu, for their valuable comments.

\section{Ethical approval}

This article does not contain any studies with human participants performed by any of the authors.

\section{Funding Statement}

This research did not receive any specific grant from funding agencies in the public, commercial, or not-for-profit sectors.

\section{Competing Interests}

The authors have declared that no competing interest exists.

\section{References}

1. Vilgelm AE, Pawlikowski JS, Liu Y, et al. Mdm2 and aurora kinase a inhibitors synergize to block melanoma growth by driving apoptosis and immune clearance of tumor cells. Cancer Res. 2015; 75:181-193.

2. Zhou N, Singh K, Mir MC, et al. The investigational Aurora kinase A inhibitor MLN8237 induces defects in cell viability and cell-cycle progression in malignant bladder cancer cells in vitro and in vivo. Clin Cancer Res. 2013; 19:1717-1728.

3. Katsha A, Soutto M, Sehdev V, et al. Aurora kinase A promotes inflammation and tumorigenesis in mice and human gastric neoplasia. Gastroenterology. 2013; 145:1312-1322.

4. Bornschein J, Nielitz J, Drozdov I, et al. Expression of aurora kinase A correlates with the Wnt-modulator RACGAP1 in gastric cancer. Cancer Med. 2016; 5:516-526.

5. Liu X, Li Z, Song $\mathrm{Y}$, et al. AURKA induces EMT by regulating histone modification through Wnt/beta-catenin and PI3K/Akt signaling pathway in gastric cancer. Oncotarget. 2016, 7:33152-33164.

6. Sherwood V. WNT signaling: an emerging mediator of cancer cell metabolism. Mol Cell Biol. 2015; 35:2-10.

7. Cong N, Du P, Zhang A, et al. Downregulated microRNA-200a promotes EMT and tumor growth through the wnt/beta-catenin pathway by targeting the E-cadherin repressors ZEB1/ZEB2 in gastric adenocarcinoma. Oncol Rep. 2013; 29:1579-1587.

8. Kimura MT, Mori T, Conroy J, et al. Two functional coding single nucleotide polymorphisms in STK15 (aurora-a) coordinately increase esophageal cancer risk. Cancer res. 2005; 65:3548-3554.

9. Hienonen T, Salovaara r, Mecklin JP, et al. Preferential amplification of aUrKa 91a (Ile31) in familial colorectal cancers. Int J Cancer. 2006; 118:505-508.

10. Gu J, Gong Y, Huang M, et al. Polymorphisms of STK15 (aurora-a) gene and lung cancer risk in Caucasians. Carcinogenesis. 2007; 28: 350-355.

11. Guénard F, labrie $Y$, Ouellette $G$, et al. Genetic sequence variations of BrCa1-interacting genes aUrKa, BaP1, BarD1 and DHX9 in French Canadian families with high risk of breast cancer. J Hum Genet. 2009; 54:152-161.

12. Egan KM, Newcomb PA, Ambrosone CB, et al. STK15 polymorphism and breast cancer risk in a population-based study. Carcinogenesis. 2004; 25: 21492153.

13. Risk M-GCoGSfMHTRBC. Polymorphisms in the BRCA1 and ABCB1 genes modulate menopausal hormone therapy associated breast cancer risk in postmenopausal women. Breast Cancer Res Treat. 2010; 120: 727-736.

14. Dicioccio RA, Song $\mathrm{H}$, Waterfall $\mathrm{C}$, et al. STK15 polymorphisms and association with risk of invasive ovarian cancer. Cancer Epidemiol Biomarkers Prev. 2004; 13: 1589-1594.

15. Lo YL, Yu JC, Chen ST, et al. Breast cancer risk associated with genotypic polymorphism of the mitosis-regulating gene AuroraA/STK15/BTAK. Int J Cancer. 2005; 115: 276-283.

16. Dai Q, Cai QY, Shu XO, et al. Synergistic effects of STK15 gene polymorphisms and endogenous estrogen exposure in the risk of breast cancer. Cancer Epidemiol Biomarkers Prev. 2004; 13: 2065-2070.

17. Ju H, Cho H, Kim YS, et al. Functional polymorphism 57Val.Ile of aurora kinase A associated with increased risk of gastric cancer progression. Cancer Lett. 2006; 242: 273-279.

18. Ye Y, Yang H, Grossman HB, et al. Genetic variants in cell cycle control pathway confer susceptibility to bladder cancer. Cancer. 2008; 112: 2467-2474.

19. Wang W, Spitz MR, Yang $\mathrm{H}$, et al. Genetic variants in cell cycle control pathway confer susceptibility to lung cancer. Clin Cancer Res. 2007; 13: 59745981.

20. Milam MR, Gu J, Yang H, et al. STK15 F31I polymorphism is associated with increased uterine cancer risk: a pilot study. Gynecol Oncol. 2007; 107: 71-74.

21. Cox DG, Hankinson SE, Hunter DJ. Polymorphisms of the AURKA (STK15/Aurora Kinase) Gene and Breast Cancer Risk (United States). Cancer Causes Control. 2006; 17: 81-83.

22. Dogan I, Ekmekci A, Yurdakul AS, et al. Polymorphisms in the aurora-A gene is not associated with lung cancer in the Turkish population. DNA Cell Biol. 2008; 27: 443-448.

23. Chen J, Sen S, Amos CI, et al. Association between Aurora-A kinase polymorphisms and age of onset of hereditary nonpolyposis colorectal cancer in a Caucasian population. Mol Carcinog. 2007; 46: 249-256.

24. LAI T.X. STK15 Phe31Ile and Val57Ile Polymorphisms Increase the Risk of Gastrointestinal Cancer. Sains Malaysiana. 2017; 47: 141-148 
25. Chong E T J , Goh L P W , See E U H , et al. Association of CYP2E1, STK15 and XRCC1 Polymorphisms with Risk of Breast Cancer in Malaysian Women. Asian Pacific Journal of Cancer Prevention Apjcp. 2016; 17:647.

26. Bao Z, Lu L, Liu X, et al. Association between the functional polymorphism Ile31Phe in the AURKA gene and susceptibility of hepatocellular carcinoma in chronic hepatitis B virus carriers. Oncotarget. 2017; 38:381.

27. Chou $\mathrm{C} \mathrm{H}$, Chou Y E, Chuang C Y, et al. Combined effect of genetic polymorphisms of AURKA and environmental factors on oral cancer development in Taiwan. Plos One. 2017; 12:e171583.

28. Chia-Hung Huang, Chih-Jung Chen, Pei-Ni Chen. Impacts of AURKA Genetic Polymorphism on Urothelial Cell Carcinoma Development. Journal of Cancer. 2019; 10:1370-1374

29. Chao Huang, Lili Wang, Hong guang Song \& Cun gang Wu. Interactive effects of AURKA polymorphisms with smoking on the susceptibility of oral cancer. Artificial Cells, Nanomedicine, and Biotechnology. 2019; 47: 2333-2337.

30. Tang W, Qiu H, Jiang H, et al. Aurora-A V57I (rs1047972) Polymorphism and Cancer Susceptibility: A Meta-Analysis Involving 27,269 Subjects. PLoS ONE. 2014; 9:e90328. 